\title{
Erratum: "Algorithms for Solving Atomic Structures of Nanodimensional Clusters in Single Crystals Based on X-ray and Neutron Diffuse Scattering Data" [Crystallogr. Rep. 49, 863 (2004)]
}

N. M. Andrushevskiǔ, B. M. Shchedrin, and V. I. Simonov

On page 865 in the right column the sentence in lines 12-14 from the bottom should read "Therefore series (7) is the superposition function $\hat{\rho}(x)$ with the period $T=L / n$ ". 